\title{
ENFOQUES PREDOMINANTES EN EL DESARROLLO COMUNITARIO EN LATINOAMÉRICA ENTRE 2009 Y 2019
}

\author{
Luis Ernesto Paz Enrique ${ }^{1}$ \\ https://orcid.org/0000-0001-9214-3057 \\ Annia Martínez Massip ${ }^{2}$ \\ https://orcid.org/0000-0001-8137-0027
}

\begin{abstract}
RESUMEN
Las categorías de desarrollo rural y agrario han sido recurrentes en las investigaciones realizadas en Latinoamérica por la importancia que tienen las mismas en la región. El desarrollo comunitario supone la identificación de capitales en el sentido que propone Pierre Bourdieu (1976). En América Latina a pesar de la diversidad de las economías muchos países son agrícolas y poco industrializados. La principal forma de obtención de riquezas es la agricultura. Se planteó como objetivo describir el enfoque rural y agrario en el desarrollo comunitario en Latinoamérica. El presente estudio es de tipo explicativo-analítico con aporte teórico. Clasifica como longitudinal-retrospectivo al analizar el período comprendido entre 2009 y 2019. Para la obtención de resultados se emplearon métodos en el nivel teórico y empírico, fundamentalmente el método bibliométrico. Se analizaron un total de 1006 artículos científicos publicados en revistas indizadas en la base de datos SciELO Citation Index. Se identificó el protagonismo de estudios sobre desarrollo local con enfoque rural y agrario. Los resultados muestran que el desarrollo comunitario en Latinoamérica es predominantemente agrario en correspondencia con categorías relativas a la soberanía alimentaria y a los territorios rurales.
\end{abstract}

Palabras clave: desarrollo rural, desarrollo agrario, desarrollo local, desarrollo comunitario, Latinoamérica.

Enfoques predominantes no desenvolvimento comunitário em América Latina entre 2009 e 2019.

\section{RESUMO}

As categorias de desenvolvimento rural e agrário tem sido recorrentes em investigações realizadas em América Latina pela importância que têm as mesmas na região. O desenvolvimento comunitário supõe a identificação de capitais no sentido que propõe Pierre Bourdieu (1976). Em América Latina apesar da diversidade das economias, muitos países são agrícolas e pouco industrializados. A principal forma de obtenção de riquezas é a agricultura. Se formulou como objetivo, descrever o enfoque rural e agrário no desenvolvimento comunitário em América Latina. O presente estudo é de tipo explicativo-analítico com contribuição teórica. Classifica como longitudinal-retrospetivo ao analisar o período compreendido entre 2009 e 2019. Para a obtenção de resultados se utilizaram métodos de níveis teóricos e empíricos, fundamentalmente o método bibliométrico. Se analisaram um total de 1006 artigos científicos publicados em revistas indexadas na base de dados SciELO Citation Index. Se identificou o protagonismo de estudos sobre desenvolvimento local com enfoque rural e agrário. Os resultados mostram que o desenvolvimento comunitário em América Latina é predominantemente agrário em correspondência com categorias relativas à soberania alimentar e aos territórios rurais. Palavras Chave: desenvolvimento rural, desenvolvimento agrário, desenvolvimento local, desenvolvimento comunitário, América Latina.

Predominant approaches in the community development in Latin America from 2009 to 2019.

\section{ABSTRACT}

The categories of rural and agrarian development have been recurrent in the investigations carried out in Latin America due to their importance in the region. The community development involves the identification of capitals in the sense proposed by Pierre Bourdieu (1976). In Latin America, despite the diversity of the economies, many countries are agricultural and little industrialized. The main way of obtaining wealth is agriculture. The objective was to describe the rural and agrarian approach of the community development in Latin America. The present study is an explanatoryanalytical type with theoretical contribution. It is classified as longitudinal-retrospective when analyzing the period between 2009 and 2019. To obtain results, were used methods at theoretical and empirical level, mainly the bibliometric one. A total of 1006 scientific articles published in journals indexed in the SciELO Citation Index database were analyzed. The role of studies on local development with a rural and agrarian focus was identified. The results

${ }^{1}$ Universidad Central "Marta Abreu” de Las Villas, Cuba. Correo electrónico: luisernestope@uclv.cu

${ }^{2}$ Universidad Central "Marta Abreu” de Las Villas, Cuba. Correo electrónico: massip@uclv.edu.cu 


\section{Sapientiae $|6|$}

show that the community development in Latin America is predominantly agrarian in correspondence with categories related to food sovereignty and rural territories.

Keywords: rural development, agrarian development, local development, community development, Latin America.

\section{Introducción}

Los campos científicos constituyen escenarios donde los intereses, competencias y comportamiento de poder; son manifestaciones por la apropiación o sujeción de capital por parte de los sujetos que lo componen: individuos, grupos de investigación e instituciones. La mayor parte de la actividad de los campos científicos (y su producción intelectual), se realiza dentro de la institucionalización universitaria. Lo anterior condiciona, a nivel regulatorio, los resultados y su forma de presentación. Respecto a lo anterior Pacheco (2006) establece que:

A todo campo corresponde cierto tipo de instancias y agentes encargados de la producción y reproducción, tanto de prácticas y procesos particulares como de saberes, habilidades, conocimientos y formas de representación simbólica que lo distinguen de otros campos (...). Un campo como el científico radica en su capacidad para incorporar y producir normas y valores que regulen socialmente el conjunto de procesos y productos de orden científico ( $\mathrm{p}$. 98).

En el seno de los campos se muestran las luchas y relaciones de fuerza entre los miembros que lo componen cuyo propósito es poseer o mantener algún tipo de los capitales que están en juego. En el campo científico la competencia son los propios miembros que producen dentro del campo. El aspecto anterior genera una dinámica de cierre para otros miembros que pretenden entrar al campo. En los roles de los campos cada investigador se crea su propia imagen y papel dentro del mismo, esto les permite conocer en qué lugar están y que acciones de fuerza que necesitan realizar para escalar a una mejor posición dentro del juego. La illusio ${ }^{3}$ del científico estará condicionada por el sistema de recompensas establecidas para los méritos que pretende obtener.

La Illusio se constituye en las mismas interacciones a las que da lugar creando una serie de prácticas que por su sentido pueden ser objetivadas o subjetivas. Está reforzada por las percepciones que el sistema de recompensas meritocrático lleve a aspirar al científico. La illusio se comporta como la coherencia del agente que compromete una serie sus prácticas a un campo científico determinado, es la suposición lógico-narrativa de que ciertas prácticas llevan a ciertas satisfacciones (Rodríguez, 2017). Es "la sumisión sin presiones al imperativo del desinterés" (Bourdieu, 1976, p.94). La illusio se puede leer en la dinámica de las redes formales e informales en las que esté inscrito el investigador y se dan por el entorno de su socialización.

Los campos existen y se generan a partir de los sistemas subjetivos de predisposiciones y expectativas que, los sujetos que lo constituyen o quieren constituir; han adquirido a través del tiempo. Los sistemas mencionados son conocidos como habitus. Lo anterior tiene lugar en la asimilación o internalización de la exterioridad. Las fuerzas exteriores se expresan desde de la lógica de los actores incorporados, generando continuidad y memoria colectiva. En consecuencia es estructurante y estructuradora (Bourdieu, 1993). A partir de lo anterior se generan las prácticas sociales que responden a la lógica del campo y su habitus que se adaptan a sus condiciones objetivas teniendo por resultado a lo "objetivamente válido" (p. 108). Se establece la racionalidad del campo cuyas expresiones son un "acto intencional de desciframiento" (Farías, 2010, p. 16).

La caracterización de un campo científico debe partir de la identificación de la lógica de su habitus. Además de aspectos relativos a las lógicas de la práctica y los roles, debe tomarse en consideración la colaboración en términos científicos que se dan en la práctica objetivada (las publicaciones). Las interacciones mencionadas, en ciertos contextos sociales e históricos, influyen en comportamientos, juicios y actitudes (Berger y Luckmann, 1966).

\footnotetext{
${ }^{3}$ Refiere a los reconocimientos y premios a los que pueden aspirar los sujetos del campo. Pude ser tanto capital de autoridad como reconocimiento entre los miembros de su comunidad que mayormente se gana a través de la citación como capital científico. La illusio general que los sujetos modulen su comportamiento a fin de obtener los méritos y recompensas establecidos.
} 
El término desarrollo en muchas ocasiones es acompañado de algún calificativo, que trastoca el concepto de desarrollo por sí solo. Hay dimensiones del desarrollo que van a estar dadas por la espacialidad física y la espacialidad social: entre lo macro y lo micro, lo local y lo global, la sociedad y la comunidad. A criterio de Riera, Paz, y Hernández (2018): las cualidades del desarrollo más frecuentes en la literatura científica publicada son: social, humano, sostenible, multidimensional, endógeno, ecodesarrollo, autodesarrollo, comunitario, económico, cultural, local, agrario, rural, entre otras.

Lo comunitario es la categoría del desarrollo que puede evidenciarse como relacional de las anteriores. Lo comunitario como cualidad del desarrollo deviene del concepto de comunidad. Las posiciones epistemológicas en torno a la comunidad han tenido una evolución desde lo geográfico hasta la configuración de grupos sociales que tienen una misma característica aunque no compartan el mismo espacio. Comunidad desde una concepción emancipadora constituye un grupo social donde transcurren procesos de participación y cooperación en torno a proyectos colectivos. Los miembros de una comunidad se identifican por compartir (al menos) una característica en común y que pueden ser tan amplias como el orden ideológico, la producción material, estratos sociales, actividad académica o pertenecer a grupos que por su condición sufren de algún tipo de discriminación.

El campo científico desarrollo comunitario se constituye desde un carácter interdisciplinar. La interdisciplinariedad parte del accionar de grupos de investigadores en la búsqueda de factores de unidad ya sea en el objeto, métodos o lenguaje (Paz y Caramés, 2020). El campo desarrollo comunitario parte de la interacción de varias ciencias y disciplinas. Dentro de las de mayor tradición en este tipo de estudios se encuentran las sociales, especialmente la psicología y la sociología.

Las investigaciones sociológicas cuyo objeto se centra en el desarrollo comunitario, parten de su división disciplinar. La amplia tradición de los estudios rurales y urbanos se centró en develar las relaciones sociales entre los sujetos ubicados en ambos contextos. La crisis de la institucionalización de la sociología rural el pasado siglo, ha influido en el debate actual sobre una sociología agraria o sociología de la agricultura que busca comprender los procesos sociales de la producción agrícola, la seguridad alimentaria, la innovación agropecuaria, entre otros conceptos. "En el ámbito latinoamericano se utilizan como sinónimos los términos de sociología rural y sociología agraria, sin embargo, por su origen, enfoques y métodos, son distintos" (González, 2016, p. 7).

La economía ha sido una disciplina recurrente en los estudios de desarrollo comunitario. Generalmente han abordado el impacto económico generado en ciertas comunidades a partir de la aplicación de proyectos. El enfoque economicista del desarrollo ha sido el más abordado (y privilegiado) desde las investigaciones científicas. Lo anterior muestra la tendencia de los campos a reproducir los enfoques dominantes, que le permitirán en última instancia, la aceptación por parte de los miembros del campo.

La deconstrucción epistemológica del desarrollo comunitario es muestra de su carácter interdisciplinar. La concepción sobre el desarrollo como, se conoce en la actualidad, deviene de la postguerra. Posterior al conflicto de la Segunda Guerra Mundial surge la necesidad de su conceptualización (Alonso, Pérez, Rivero, Romero, y Riera, 2004; Figueroa, 2009). Luego del enfrentamiento bélico surgen los términos que se derivan del desarrollo tales como subdesarrollo, países en vías de desarrollo, desarrollo local, desarrollo humano, desarrollo sostenible, entre otros (Boisier, 1999).

El campo científico desarrollo comunitario supone la identificación de capital en el sentido que le aporta Bourdieu (1976), actores y relaciones sociales para la obtención del mismo. Las categorías del desarrollo rural y agrario resultan muy importantes en las investigaciones que se desarrollen desde Latinoamérica por las características de la región. En América Latina a pesar de la diversidad de las economías muchos países son agrícolas y poco industrializados. La principal forma de obtención de riquezas es la agricultura, sin embargo "...el asunto de la tierra sigue sin resolverse" 
(Kay, 2016, p. 185) y “...el desarrollo en beneficio de... todos sus campesinos, es un asunto que está por inventar” (Amin, 2016, p. 227).

Se planteó como objetivo describir el enfoque rural y agrario del campo científico desarrollo comunitario en Latinoamérica. El artículo se estructura en una introducción enfocada en establecer los aspectos teóricos relativos a los campos científicos y sus manifestaciones en la literatura científica publicada. Tiene un acápite metodológico cuya función es exponer los principales métodos y herramientas que permiten la obtención de datos empíricos. Los resultados y discusión muestran que los principales enfoques del campo científico desarrollo comunitario en Latinoamérica se relacional con lo rural, agrario y local. Por último se presentan las conclusiones del estudio que constituyen generalizaciones del trabajo realizado.

\section{Metodología}

El presente estudio es de tipo explicativo-analítico con aporte teórico. Clasifica como longitudinalretrospectivo al analizar el período comprendido entre en 2009 y 2019. Se enfoca en identificar las características del campo científico desarrollo comunitario desde un análisis temático. La unidad de observación se estructura a partir de las publicaciones o documentos generados por grupos o comunidades científicas. Para la obtención de resultados se aplican métodos en los niveles teórico y empírico.

En el nivel teórico se emplean los métodos analítico-sintético y sistémico-estructural. En el nivel empírico se emplea el análisis documental clásico y fundamentalmente el método bibliométrico. Los procederes a seguir a partir del empleo del método bibliométrico se ajustaron a las siguientes pautas con referentes en estudios de Carpintero y Tortosa (1990), Spinak (1996), Machado y López (2015), Olivera, Peralta, y García. (2018).

1. Selección de las fuentes de información: la base de datos SciELO Citation Index. Esta base de datos se deriva de SciELO y cuenta con colecciones de revistas latinoamericanas que han tenido un mayor impacto dentro de las restantes. La base de datos está incluida en la Web of Science y es la única enteramente latinoamericana. Contiene literatura científica publicada en las revistas de mayor calidad de la región. Se obtuvo una base de datos con un total de 2663 registros.

2. Dimensión espacial y temporal: se enmarcó en la producción científica comprendida en el período entre el 2009 y 2019. La selección del período de tiempo permite identificar las tendencias del campo científico en la última década.

3. Confección de las bases de datos: se importaron los registros de la base de datos a partir de la búsqueda por tema "desarrollo comunitario" y sus equivalentes en inglés y portugués.

4. Estrategia de búsqueda: para localizar la producción científica del campo del conocimiento desarrollo comunitario se trazó como estrategia de búsqueda: TEMA: (desarrollo comunitario/ community develpment/ desenvolvimento da comunidade) AND year_cluster: ("2009" OR "2010" OR "2011" OR 2012" OR "2013" OR "2014" OR "2015" OR "2016" OR "2017" OR "2018" OR "2019")

5. Normalización de los datos: en un primer momento se eliminan todos los duplicados existentes. Luego se procede a borrar los documentos que no tenían relación con el desarrollo comunitario. Se verifica en cuanto a la autoría, si eran los mismos autores, porque en algunos casos había autores con el mismo apellido, comenzaban los nombres con la misma inicial y eran autores diferentes. En otros casos unos tenían dos apellidos y más, y en algunas ocasiones no tenían nombre. En las palabras clave existían errores en cuanto a singulares y plurales. Luego de la normalización la base de datos quedó con un total de 1006 registros bibliográficos.

6. Procesamiento de los datos: el cálculo de los indicadores seleccionados y su representación se realizó mediante el programa Microsoft Excel de Microsoft Office 2007, además del EndNote X7. 
7. Los indicadores seleccionados atendiendo a la producción científica fueron los siguientes:

- Indicador productividad por áreas temáticas: consiste en identificar las disciplinas o áreas de la ciencia a las que tributan los estudios publicados bajo campo científico desarrollo comunitario. Los resultados se visualizan a través de un ranking de forma descendente y un gráfico de barras con el apoyo del software microsoft excel.

- Indicador mapa de palabras: constituye una forma de visualizar los resultados a partir de la densidad o la frecuencia o preponderancia de unos términos sobre otros. Se realiza a partir de la visualización de los resultados en una nube de etiquetas con el objetivo de mostrar cuáles son los términos más frecuentes en los resúmenes, títulos y palabras clave. Para la realización de la nube de etiquetas en los resúmenes se empleó el sitio web: https://worditout.com/ y para títulos y palabras clave los softwares VOSviewer_1.4.0. y Net Draw.

El resumen ${ }^{4}$ o síntesis por lo general es un requerimiento en las comunicaciones que se socializan en revistas científicas. En la actualidad el resumen más empleado es el estructurado, por lo que se puede conocer el contenido y hallazgos de las investigaciones sin necesidad de hacer una lectura completa. La representación a través de mapas de palabras ${ }^{5}$ permite visualizar la preponderancia de temáticas y enfoques metodológicos.

- Indicador palabras clave más productivas: se define como la cantidad de veces que una misma palabra clave es empleada para indizar artículos dentro de la muestra analizada en cada año. Los resultados se visualizan a partir de la representación multivariada por densidad de palabras clave, esto se realiza con el apoyo del software VOSviewer.

- Indicador palabras clave más productivas por años: se empleó el indicador para analizar si en algunos años o períodos los términos asociados al desarrolllo comunitario variaron. Se muestran las principales palabras clave más utilizadas en las publicaciones científicas. Se ilustraron a partir de la combinación de gráfico de área y cronológico generado en el software Excel a partir de la elaboración de una matriz por años y las tres palabras clave más frecuentes.

Atendiendo a la colaboración los indicadores fueron:

- Indicador coocurrencia de palabras clave: las palabras clave son un requerimiento para las principales comunicaciones científicas que se socializan en revistas, por lo que constituyen descriptores $^{6}$. Muestran las principales temáticas que aborda una comunicación. Por lo general las publicaciones solicitan a los investigadores que extraigan las palabras clave de tesauros $^{7}$ donde se localiza el lenguaje de las ciencias. En las palabras clave radican los principales contenidos que aborda un estudio. La representación multivariada en frecuencia de palabras clave muestra los principales nodos en que se aborda el desarrollo comunitario. La representación mediante el análisis de redes sociales muestra los términos centrales y la coocurrencia en las que aparecen los términos en las comunicaciones científicas.

- Indicador centralidad de palabras clave: la centralidad es una prueba estadística que se aplica a unidades de observación que poseen múltiples relaciones. Los resultados de la

\footnotetext{
${ }^{4}$ Cabe destacar que en las comunicaciones científicas existen 3 tipos de resúmenes: 1) informativo (hasta 150 palabras, de carácter descriptivo sobre el propósito del estudio), 2) indicativo (hasta 200 palabras, contiene los objetivos y principales resultados) y 3 ) estructurado (de 250 palabras hasta 300. Se compone por la problematización, objetivos, metodología empleada, principales resultados y conclusiones).

${ }^{5}$ Los mapas de palabras son una representación a partir de densidad, donde se muestra la ocurrencia de términos con mayor frecuencia en una o varias fuentes.

${ }^{6}$ Los descriptores, a menudo también denominados metadatos, pueden ser temáticos (asunto o tema), cronológicos (mención de tiempo y fechas), topográficos (mención de lugares) y onomásticos (referente a personas).

${ }^{7}$ Los tesauros son una estructura que contiene el lenguaje controlado (oficializado) de una ciencia. Aunque en las ciencias naturales, técnicas y exactas el uso de tesauros es muy estricto (por ejemplo para los nombres científicos que se emplean en la biología y la agronomía); para las ciencias sociales su uso es muy variado. Se recomienda para estas ciencias el uso del tesauro de la UNESCO.
} 
centralidad permiten establecer cuáles categorías constituyen nodos centrales atendiendo al campo. Pala la visualización de los resultados se empleó el software NetDraw.

Las técnicas empleadas que facilitan la recogida de información son la revisión de documentos y el análisis de redes sociales.

\section{Resultados y discusión}

El análisis temático de campos científicos incluye disciplinas, títulos, términos empleados en resúmenes de las publicaciones y palabras clave. Son los elementos que permiten identificar el área, asunto o tema que abordan las publicaciones correspondientes al campo científico desarrollo comunitario en Latinoamérica. En la Figura 1 se muestran las áreas temáticas más productivas del campo.

Figura 1: áreas temáticas más productivas del campo

\section{Areas temáticas más productivas del campo}

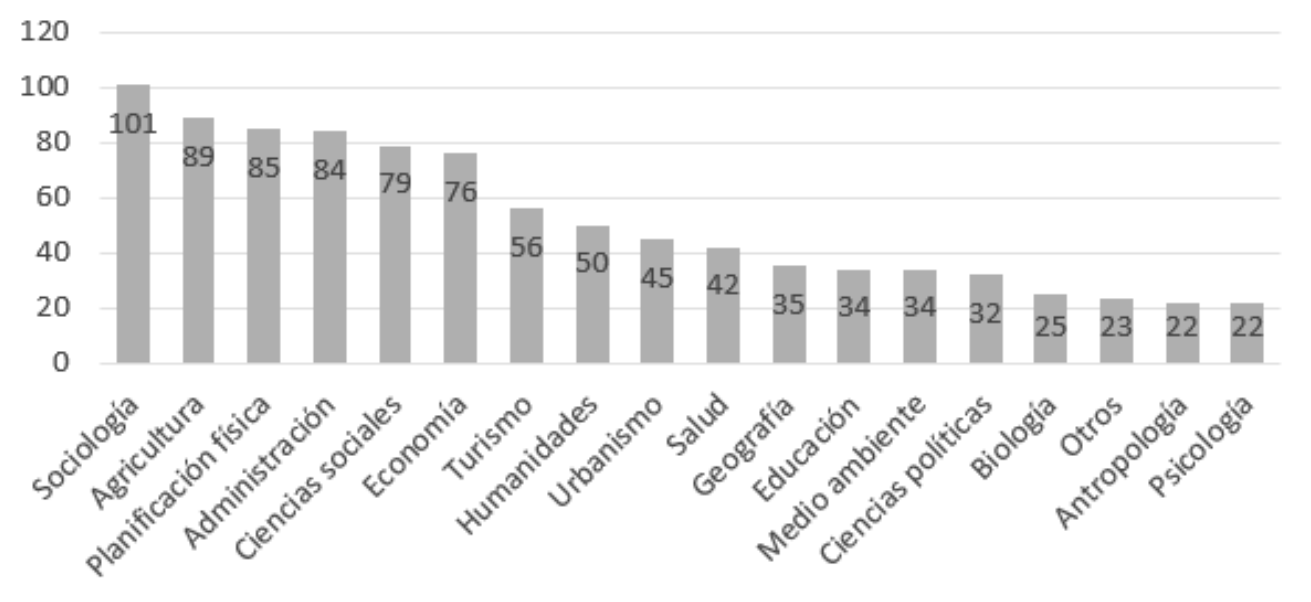

Fuente: elaboración propia a partir del uso del software Excel 2010.

Atendiendo a las áreas temáticas más productivas, la Sociología tuvo mayor protagonismo, reportando 101 artículos. La sociología estudia los fenómenos colectivos producidos por la actividad social de los seres humanos, dentro del contexto histórico-cultural en el que se encuentran inmersos. La sociología del desarrollo es una disciplina de la ciencia mencionada, que analiza los fenómenos concernientes al devenir y tránsito superior de individuos y comunidades (Gunder, 1969). El predominio de estudios sobre desarrollo comunitario a partir de postulados sociológicos permite abordar el campo científico tomando como centro los procesos y determinantes sociales que se realizan en contextos particulares.

El hecho de que la sociología sea el área temática que aborda el desarrollo comunitario con mayor regularidad puede clasificarse de positivo. Las publicaciones correspondientes a esta temática se centraron en estudios de pobreza, desigualdades y migración. Se evidenció un predominio de estudios de desarrollo local en contextos rurales, específicamente relacionados con la alimentación y la agricultura familiar.

En correspondencia al criterio anterior se identifica que la agricultura es la segunda área temática con 89 publicaciones. La soberanía alimentaria, el autoabastecimiento de las localidades es objeto del desarrollo comunitario enfocado en el contexto rural. La agricultura resulta ser el renglón económico esencial en muchas localidades latinoamericanas. La agricultura en la economía latinoamericana es un sector productivo de suma importancia en la mayoría de los países. 
Por ello, no es casual que:

En el ámbito académico latinoamericano la sociología rural aparece más consolidada, tanto por su presencia en las universidades, como por la existencia de centros de investigación, revistas especializadas, organizaciones de profesionales. Las instituciones académicas, han tenido una importante función en el desarrollo de la sociología agraria (González, 2016, p. 7).

La acumulación sostenida del problema agrario en América Latina desde la anterior centuria ha agudizado, en la última década, la pobreza rural en detrimento del desarrollo comunitario y local. De ahí que, se denota un incremento de de los estudios en sociología rural o agraria a nivel de pregrado y postgrado, frente a un contexto de políticas antiagrarias incapaces de enfrentar la desigualdad social y el atraso económico en las ruralidades.

Si se valora el área de la administración y la economía como una misma por su similitud y finalidad, suman un total de 160 publicaciones. La planificación física ocupa una tercera posición con 85 artículos. La planificación física puede considerarse como mediadora entre los estudios urbanísticos y de reordenamiento territorial, pero con un enfoque económico dominante. Los aspectos anteriores permiten establecer que durante la última década el desarrollo comunitario en Latinoamérica tuvo y tiene un enfoque predominantemente económico. El resultado obtenido no resulta contradictorio a la lógica del campo debido a que la reproducción del enfoque es una vía para su legitimidad.

El análisis de los elementos formales de la comunicación científica escrita permite establecer temáticas y enfoques. El título de las publicaciones es un argumento breve que debe resumir el contenido del documento en su totalidad. Los términos más frecuentes en los títulos de las publicaciones del campo científico desarrollo comunitario se observan en la Figura 2.

Figura 2: palabras más frecuentes en los títulos de las publicaciones del campo científico desarrollo comunitario en Latinoamérica

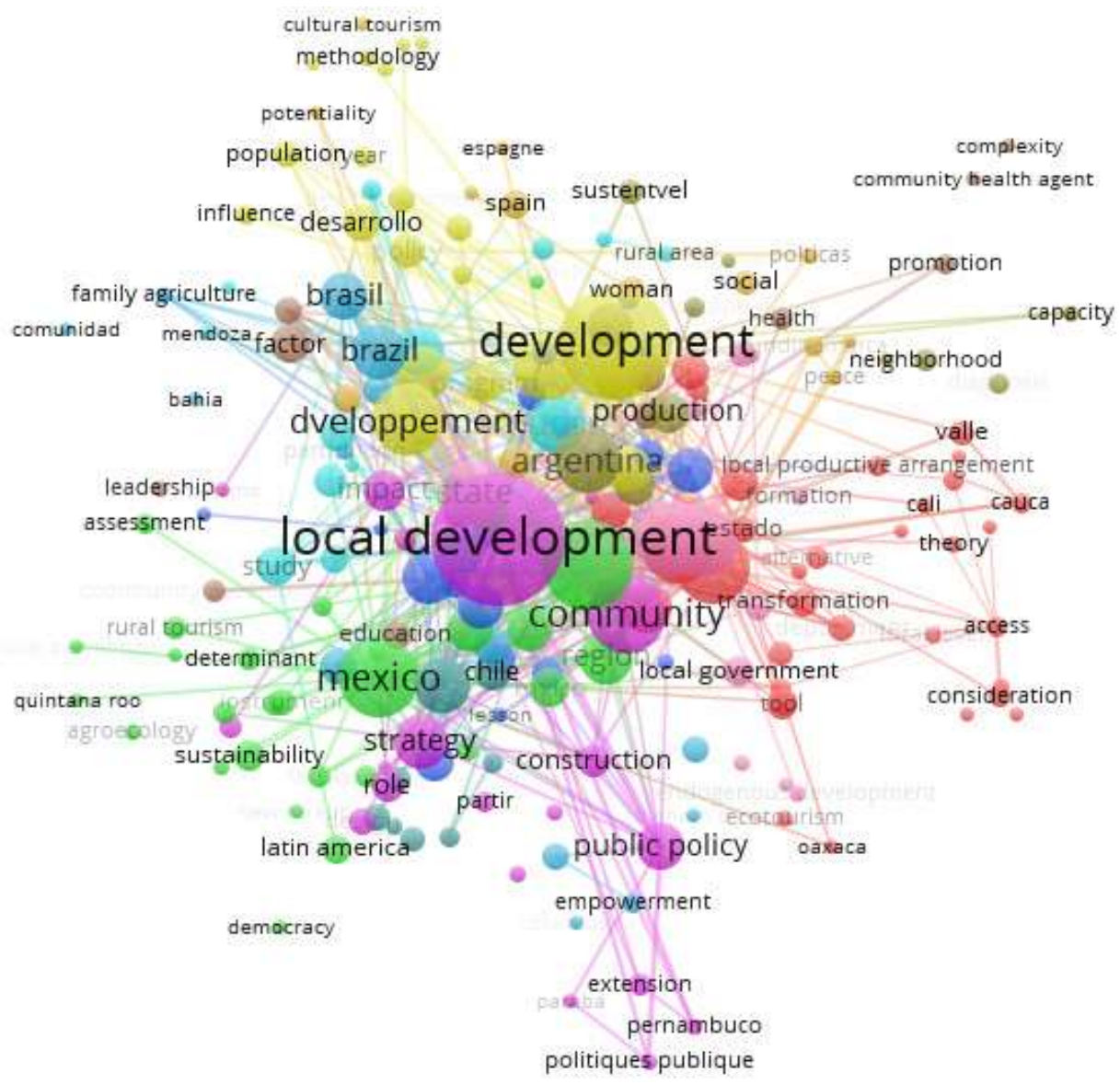

Fuente: elaboración propia a partir del uso del software VOSviewer_1.4.0. 
Los resultados obtenidos se corresponden con un análisis similar realizado a los resúmenes de las publicaciones del campo. Los resúmenes de los artículos deben ser estructurados: alrededor de 250 palabras, deben contener la estructura metodológica del estudio, los principales resultados y conclusiones. Lo anterior posibilita visualizar una diversidad de aspectos que se abordan en las publicaciones del campo. La Figura 3 muestra un mapa de palabras con los términos más frecuentes en los resúmenes de las publicaciones del campo.

Figura 3: mapa de palabras más frecuentes en los resúmenes de las publicaciones del campo científico desarrollo comunitario en Latinoamérica

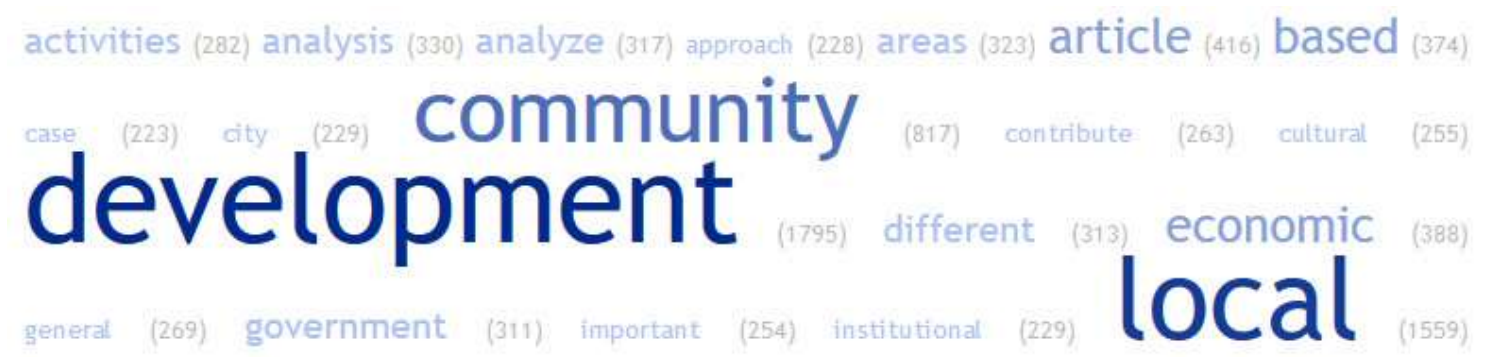

management (358) model (301) municipalities (331) objective (251) organizations (261) paper (267)

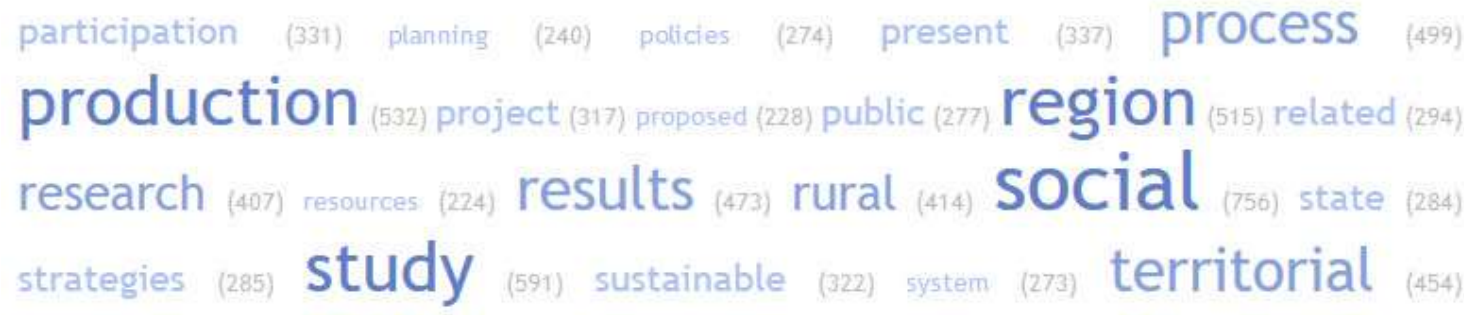

\section{tourism (371) work (256)}

Fuente: elaboración propia a partir del uso de sitio https://worditout.com/.

En ambos análisis el término con mayor regularidad fue desarrollo local. Las investigaciones que fueron realizadas bajo este término estuvieron asociadas a varios estudios de caso efectuados en comunidades rurales, pueblos originarios y cultura popular y tradicional. El término con mayor frecuencia luego de desarrollo local fue comunidad (community). El desarrollo comunitario es un área donde pueden confluir otras categorías del desarrollo dentro de ellas lo local que en Latinoamérica tiene como escenario fundamental lo rural y agrario. A criterio de Martínez (2016) la comunidad es siempre un grupo humano complejo que de alguna manera comparte, con diverso condicionamiento, la participación en torno a tareas comunes, establece relaciones de cooperación y propicia determinado grado de implicación de las personas que integran dicho grupo.

Se reflejan otros términos como gobierno local, vecindad, empoderamiento y liderazgo. De lo anterior puede ratificarse la relación del campo científico desarrollo comunitario con los gobiernos locales: las iniciativas locales de desarrollo relativas a la innovación y a la articulación de proyectos, deben estar respaldadas por los gobiernos locales. La particularidad del desarrollo comunitario en Latinoamérica es que hace énfasis (al menos es lo que se refleja en el nivel científico) en el empoderamiento de los actores comunitarios y la participación de la vecindad como beneficiaria de proyectos articuladores. Del empoderamiento de los actores surgen líderes informales, líderes comunitarios que juegan un papel fundamental.

Otro de los términos destacados es región en referencia al espacio que ocupa la comunidad. En América Latina se le atribuye importancia a la identidad de lo local, fundamentalmente en etnias y poblacionales con tradiciones culturales fuertemente arraigadas. La extinción de ciertas localidades y con ellas su cultura tradicional es resultado de los siglos de explotación y colonización, por lo 
que la recuperación de lo regional es tarea de primer orden en Latinoamérica. El criterio anterior se apoya en que el término ciudad (city) es uno de los menos frecuentes.

Sustentable es un término que aparece con frecuencia en títulos y resúmenes del campo. Desarrollo sustentable y desarrollo económico son dos de las categorías que se le asignan al desarrollo. Anteriormente se plantea que los estudios del campo son fundamentalmente con rasgo económico, sin embargo, el desarrollo sustentable debe complementar el desarrollo económico, de forma que el progreso de la economía sea capaz de mantenerse en el tiempo sin causar afectaciones al medio ambiente.

El análisis de las palabras clave permite identificar subtemáticas o subcategorías en el campo científico desarrollo comunitario en Latinoamérica. Las palabras clave son términos de indización que permiten posteriormente la recuperación del documento por otros investigadores. Generalmente se elaboran con lenguaje controlado, por lo que se necesita el uso de tesauros. Lo anterior garantiza una estandarización en las investigaciones para declarar este aspecto dentro de los artículos. La Figura 4 muestra las palabras clave más productivas del campo.

Figura 4: palabras clave más frecuentes en las publicaciones del campo científico desarrollo comunitario en Latinoamérica

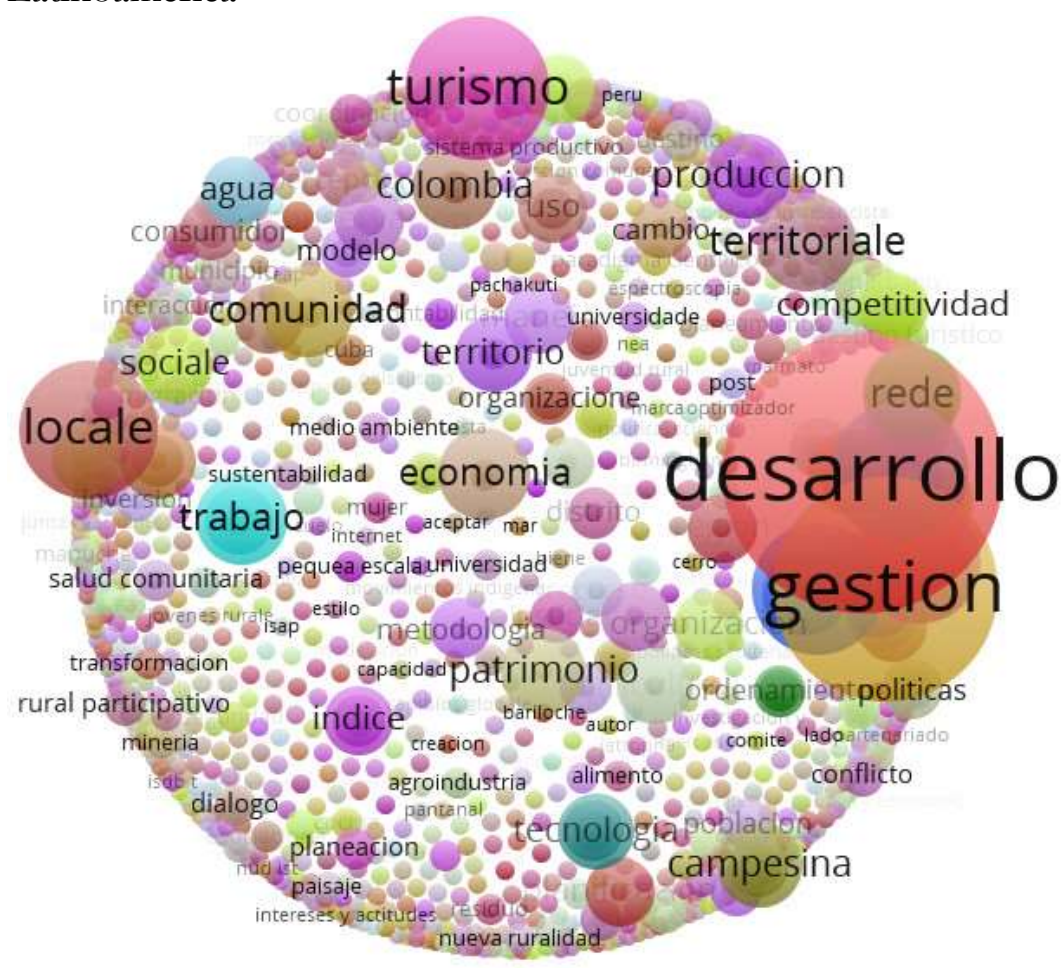

Fuente: elaboración propia a partir del uso del software VOSviewer_1.4.0.

Durante el período analizado las palabras clave más frecuentes se correspondieron al enfoque rural y agrario que predomina en la región. La palabra clave más empleada fue desarrollo local, utilizada en 195 ocasiones. Lo anterior es muestra de que el desarrollo comunitario se utiliza como escenario y no como contexto. El campo científico desarrollo comunitario en Latinoamérica está dominado por la subtemática desarrollo local que se enfoca fundamentalmente en estudios rurales y agrarios. Desarrollo rural como término fue utilizado en 45 publicaciones siendo el segundo más empleado. También es recurrente la palabra gestión territorial o gestión del desarrollo.

La palabra clave turismo es frecuente, el turismo comunitario y el turismo cultural es una alternativa popular en Latinoamérica por la existencia de comunidades originarias. La contribución del turismo al desarrollo de la población debe lograr con esta actividad no solo crecimiento económico, sino también un motor de progreso para la población, ya sea en entornos rurales, 
urbanos y silvestres, entre otros (Burgos, 2016). El turismo comunitario en la región se enfoca en la explotación de recursos naturales con singular belleza (como playas, biodiversidad biológica, ríos, lagos, entre otros), la muestra de la cotidianidad de comunidades que tienen formas de vida arraigadas a sus ancestros y la cultura popular enfocada fundamentalmente en platos culinarios y la artesanía.

Lo anterior puede constituir una preocupación para la sostenibilidad de los proyectos de desarrollo basados en el turismo. El foráneo por lo general llega a depredar los sitios que visita: sustrae especies autóctonas, introduce otras, contamina los recursos naturales e impone su cultura que puede ser poco a poco asimiladas por las comunidades. Otro aspecto que debe ser valorado es que por lo general dentro de las comunidades no todos los individuos se dedican a la actividad turística. El desarrollo desigual de unos miembros con respecto a otros a partir de su poder adquisitivo puede generar problemáticas dentro de la comunidad.

En las áreas rurales el acceso a los bienes y servicios es desigual. La agricultura constituye una vía para el desarrollo de estas regiones. En América Latina a pesar de la diversidad de las economías, muchos países son eminentemente agrícolas y poco industrializados. El desarrollo agrario es su vía fundamental para la generación de recursos (Kay, 2009; Organización de las Naciones Unidas Para la Alimentación y la Agricultura, 2012; Olson y Fahey, 2011).

Durante el período analizado se evidenciaron algunas diferencias en cuanto al predominio de unas palabras clave sobre otras en el período 2009 y 2019. La producción de palabras clave por años se observa en la Figura 5.

Figura 5: palabras claves por años en las publicaciones del campo científico desarrollo comunitario en Latinoamérica

\section{Palabras clave por años más frecuentes en el campo}

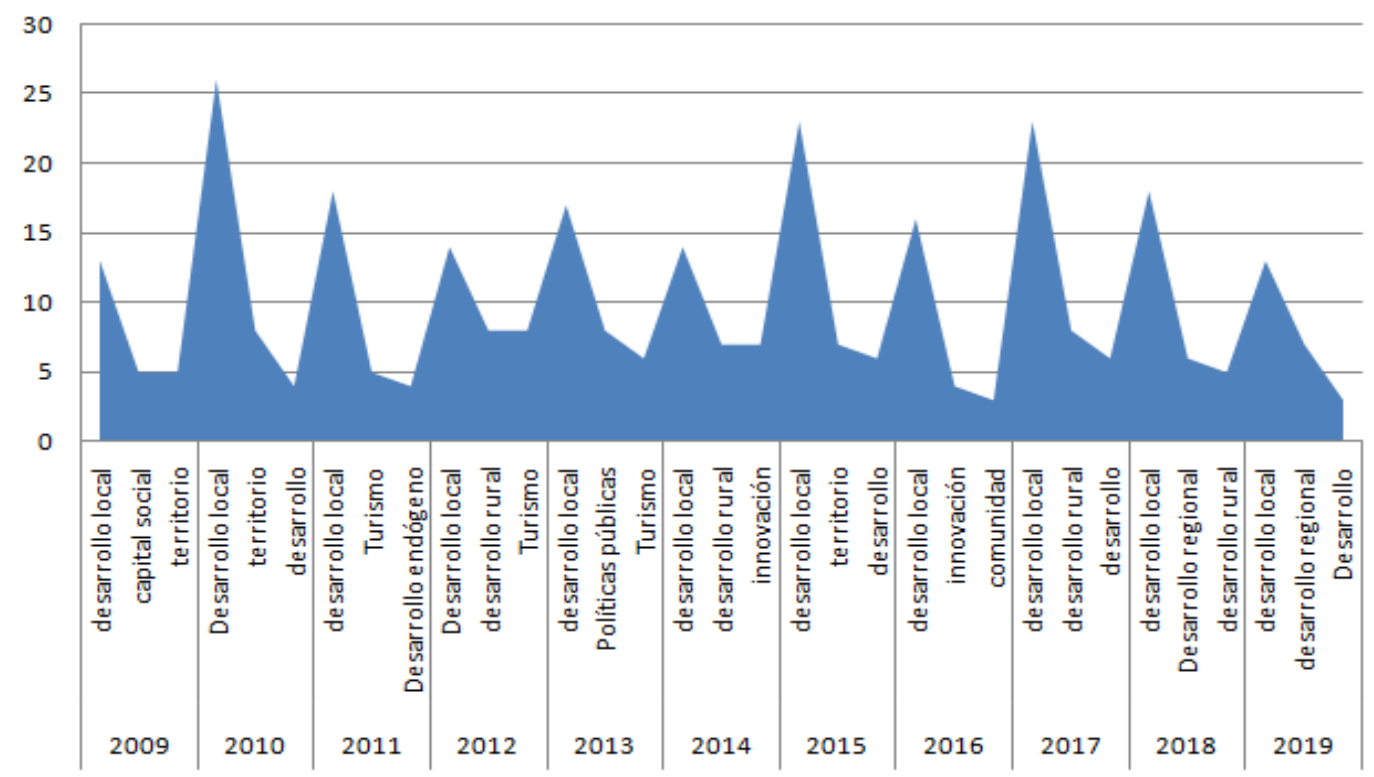

Fuente: elaboración propia a partir del uso del software Excel 2010.

El término desarrollo local se mantuvo con mayor frecuencia durante todo el período analizado. En la distribución por años se pueden visualizar otras palabras clave como desarrollo endógeno, capital social, políticas públicas, turismo, innovación y comunidad. En la muestra seleccionada frecuentemente se observa que los estudios realizados hacen referencia a lo local para situar un marco geográfico en investigaciones de otras áreas que no abordan la conceptualización de desarrollo comunitario en sí mismo. Se evidencia la ausencia de estudios con aporte teórico y 
metodológico, predominado estudios de caso como forma de socializar experiencias en torno al desarrollo comunitario.

Se realiza un análisis de la coocurrencia de palabras clave de los términos que coocurren 10 o más veces en la producción científica del campo desarrollo comunitario en Latinoamérica. Los resultados obtenidos se muestran en la Figura 6.

Figura 6: coocurrencia de palabras clave de los términos que coocurren 10 o más veces en la producción científica del campo desarrollo comunitario en Latinoamérica

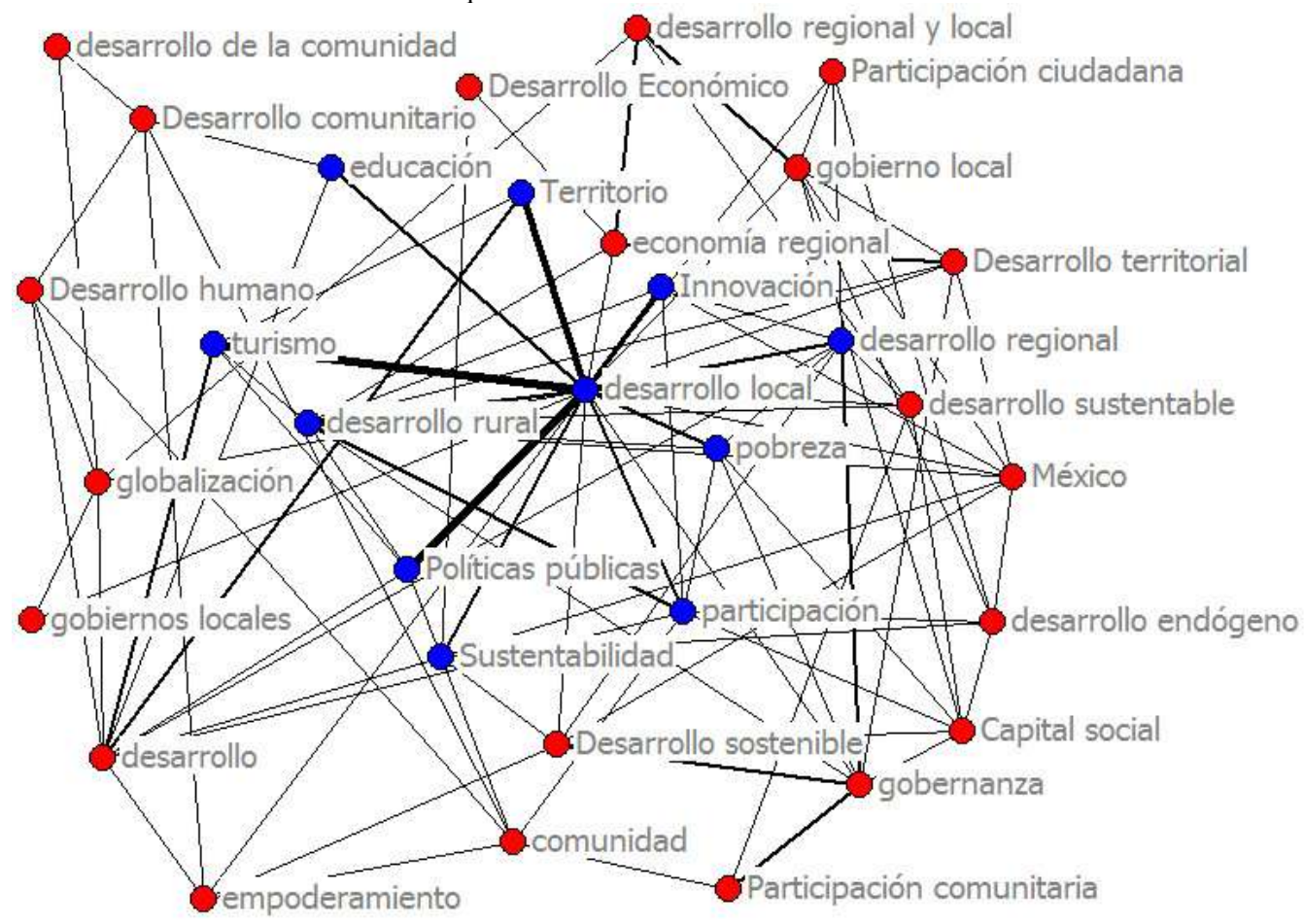

Fuente: elaboración propia a partir del uso del software Net Draw.

$\mathrm{Al}$ resultado obtenido se le aplicó una prueba de centralidad para identificar aquellas palabras clave que mayores relaciones tienen con otras. El análisis realizado se muestra en la Figura 7. 
Figura 7: prueba de centralidad aplicada a la coocurrecia de palabras claves del campo desarrollo comunitario en Latinoamérica

\begin{tabular}{|c|c|c|c|}
\hline & $\begin{array}{r}1 \\
\text { Degree }\end{array}$ & NrmDegree & Share \\
\hline desarrollo local & 88.000 & 18.295 & 0.208 \\
\hline turismo & 21.000 & 4.366 & 0.050 \\
\hline desarrollo rural & 20.000 & 4.158 & 0.047 \\
\hline desarrollo & 19.000 & 3.950 & 0.045 \\
\hline Politicas públicas & 19.000 & 3.950 & 0.045 \\
\hline gobernanza & 18.000 & 3.742 & 0.042 \\
\hline participación & 15.000 & 3.119 & 0.035 \\
\hline Territorio & 15.000 & 3.119 & 0.035 \\
\hline desarrollo regional & 14.000 & 2. 911 & 0.033 \\
\hline Innovación & 13.000 & 2.703 & 0.031 \\
\hline Sustentabilidad & 13.000 & 2.703 & 0.031 \\
\hline Desarrollo sostenible & 12.000 & 2.495 & 0.028 \\
\hline economía regional & 12.000 & 2. 495 & 0.028 \\
\hline Desarrollo territorial & 11.000 & 2.287 & 0.026 \\
\hline gobierno local & 10.000 & 2.079 & 0.024 \\
\hline México & 10.000 & 2.079 & 0.024 \\
\hline Capital social & 9.000 & 1. 871 & 0.021 \\
\hline empoderamiento & 9.000 & 1.871 & 0.021 \\
\hline pobreza & 9.000 & 1.871 & 0.021 \\
\hline desarrollo regional y local & 9.000 & 1.871 & 0.021 \\
\hline comunidad & 9.000 & 1.871 & 0.021 \\
\hline globalización & 9.000 & 1.871 & 0.021 \\
\hline desarrollo endógeno & 8.000 & $1.66 \overline{3}$ & 0.019 \\
\hline educación & 7.000 & 1. 455 & 0.017 \\
\hline Desarrollo comunitario & 6.000 & 1. 247 & 0.014 \\
\hline
\end{tabular}

Fuente: elaboración propia a partir del uso del software Net Draw.

Las relaciones de coocurrencia entre palabras clave muestran el predominio del término desarrollo local con otros. Lo anterior se ratifica en la prueba de centralidad realizada. Las relaciones del término desarrollo local con otros se sustenta en que los estudios se centran en contextos particulares para analizar las problemáticas sociales y los aspectos que limitan o están presentes en las comunidades para el logro de su desarrollo. Posteriormente aparece turismo como un segundo núcleo que se relaciona con desarrollo rural (que ocupa el tercer puesto), políticas públicas (quinto puesto) y territorio (octavo puesto). Las relaciones anteriores se sustentan en las políticas que han asumido los diversos territorios y comunidades latinoamericanas en cuanto al turismo, que es una fuente generadora de riquezas en la región. En la producción científica del campo el ecoturismo es un término recurrente y se da fundamentalmente en áreas rurales.

Los términos territorio, desarrollo rural, comunidad e innovación convergen en el desarrollo local. Las zonas rurales en Latinoamérica en su mayoría tienen un elevado nivel de pobreza de ahí la importancia de fomentar el desarrollo de políticas basadas en un turismo de poca inversión. En América Latina existen comunidades originarias y culturas tradicionales que son centro de atención para la articulación de proyectos de desarrollo. Las políticas públicas según Montoro (1997) son el diseño y la ejecución programada y estructurada de todas aquellas iniciativas adoptadas para atender una serie de necesidades. Los gobiernos locales deben optar por iniciativas que apuesten por la participación ciudadana en la confección de políticas públicas como vía idónea para el logro del bien común. Las políticas públicas deben ser elaboradas teniendo en cuenta las necesidades del territorio para favorecer el desarrollo comunitario.

La prueba de centralidad aplicada muestra que el término desarrollo comunitario aparece en la red de palabras clave en el lugar 25, con anterioridad aparecen: comunidad (lugar 21) y desarrollo endógeno (lugar 23). Aunque la prueba realizada no favorece al desarrollo comunitario atendiendo a su representación en cuanto a posición dentro del resto de los términos, no puede afirmarse que los resultados no se correspondan a aspectos que contempla el campo. Asociadas al desarrollo comunitario se identifican las siguientes subtemáticas que ocupan un lugar inferior dentro del ranking: participación, territorio, desarrollo regional, innovación, sustentabilidad, desarrollo 
sostenible, desarrollo territorial, gobierno local, capital social, empoderamiento y participación comunitaria.

\section{Conclusiones}

Las áreas temáticas con mayor presencia fueron sociología, agricultura y planificación física. La sociología dentro del campo se enfocó en estudios de pobreza, desigualdades y migración. Se evidenció un predominio de estudios de desarrollo local en contextos rurales, específicamente relacionados con la alimentación y la agricultura familiar. La agricultura como área temática se enfocó en la soberanía alimentaria, el autoabastecimiento de las localidades; aspectos que son objeto del desarrollo comunitario enfocado en el contexto rural.

La planificación física como área temática se enfocó en el reordenamiento territorial tanto rural como urbanístico. En el contexto rural tuvo como objetivo aumentar la productividad de la agricultura, por lo que la finalidad fue eminentemente económica. Los resultados arrojan que la sumatoria de los resultados del área administración y economía ubican los enfoques económicos en primer lugar. El desarrollo comunitario en Latinoamérica tuvo y tiene un enfoque predominantemente económico.

Los mapas de palabras realizados a los títulos, resúmenes y palabras clave muestran un predominio del término desarrollo local. Los resultados se corresponden a que las publicaciones estuvieron asociadas a varios estudios de caso efectuados en comunidades rurales y pueblos originarios. Se reflejan otros términos como gobierno local, vecindad, empoderamiento y liderazgo. El campo científico desarrollo comunitario tiene una gran relación con los gobiernos locales. Se destaca el término región en referencia al espacio que ocupa la comunidad. Los términos con mayor frecuencia referían a lo rural como contexto de estudio, evidenciado en que el término ciudad es uno de los menos frecuentes.

Atendiendo a las palabras clave más productivas el desarrollo local que se enfocó fundamentalmente en estudios rurales y agrarios. Desarrollo rural ocupó la segunda posición, la mayor parte de los términos fueron asociados al mismo, tal es el caso de turismo que resultó muy recurrente en la producción científica. El turismo comunitario y el turismo cultural es una alternativa popular en Latinoamérica por la existencia de comunidades originarias. El turismo comunitario o turismo rural se enfocó en la categoría de turismo de naturaleza por el contexto rural donde se realiza el mismo. Destaca lo agrario debido a que es la principal forma de subsistencia y generar riquezas en América Latina.

Atendiendo a las palabras clave más productivas por años no se evidencian diferencias significativas, sin embargo, la prueba de centralidad muestra los términos que más coocurren con otros. Desarrollo local es el término que aparece con mayor regularidad. Turismo como un segundo núcleo se relaciona con desarrollo rural, políticas públicas y territorio. Los aspectos anteriores convergen en la socialización de experiencias locales a partir de asumir políticas públicas sobre el ecoturismo como forma de generar beneficios económicos. La prueba de centralidad muestra que el término desarrollo comunitario aparece en la red de palabras clave en el lugar 25. El resultado muestra que a nivel de la comunicación científica no fue ampliamente privilegiado, sin embargo, los términos con mayor presencia reflejan que los estudios se realizaron en a las ruralidades y al ámbito agropecuario.

\section{Referencias bibliográficas}

Alonso, Joaquín; Pérez, Armando; Rivero, Ramón; Romero, Edgar y Riera, Celia Marta. (2004). Autodesarrollo Comunitario: crítica a las mediaciones sociales recurrentes para la emancipación humana. Editorial Feijóo, Cuba.

Amin, Samir. (2016). Agriculturas capitalistas o agriculturas en el capitalismo. Convergencias y diferencias. Agricultura campesina, agricultura familiar moderna. 
En: Sociología agraria. Procesos agrarios en Cuba y América Latina. Editorial Universitaria Félix Varela, Cuba.

Berger, Peter y Luckmann, Thomas. (1966). The Social Construction of Reality: a Treatise of Sociology of Knowledge. Doubleday: Estados Unidos.

Boisier, Sandro. (1999). Desarrollo (local): ¿De qué estamos hablando? CEDET, Argentina. Bourdieu, Pierre. (1976). Le champ scientifique. Actes de la recherche en sciences sociales. Vol. 2 No. 2. Francia (Pp. 88-104).

Bourdieu, Pierre. (1993). El sentido páctico. Editorial Taurus, España.

Burgos, Rosalía. (2016). El turismo comunitario como iniciativa de desarrollo local: Caso localidades de Ciudad Bolívar y Usme zona rural de Bogotá. Hallazgos. Vol. 13 No. 26. Colombia (Pp. 193-214). Doi: 10.15332/s1794-3841.2016.0026.08

Carpintero, Hilda y Tortosa, Federico. (1990). Aplicaciones de la metodología bibliométrica a la historia de Psicología: una revisión de conjunto. PPU, España.

Farías, Ignacio. (2010). Adieu à Bourdieu? Asimetrías, límites y paradojas en la noción de habitus. Convergencia, Revista de Ciencias Sociales. Vol. 54 No. 2010. México (Pp. 11-34).

Figueroa, Galia. (2009). Ciencias sociales cubanas del siglo veintiuno y el problema del desarrollo. Una fascinación contradictoria. Universidad de La Habana, Cuba.

González, Ernel. (2016). Sociología agraria: orígenes, evolución y problemas actuales. En: Sociología agraria. Procesos agrarios en Cuba y América Latina. Editorial Universitaria Félix Varela, Cuba.

Gunder, André. (1969). La sociología del desarrollo y el subdesarrollo de la sociología. Cuadernos Anagrama, España.

Kay, Cristóbal. (2009). Estudios rurales en América Latina en el periodo de globalización neoliberal: ¿una nueva ruralidad? Revista mexicana de sociología. Vol. 71 No. 4. México (Pp. 607-645).

Kay, Cristóbal. (2016). América Latina. Mirando hacia atrás: el tiempo de las reformas agrarias. En: Sociología agraria. Procesos agrarios en Cuba y América Latina. Editorial Universitaria Félix Varela, Cuba.

Machado, Manuel Osvaldo y López, Julio. (2015). Producción científica relativa a los servicios de Información de medicamentos en la Web of Science. Medicentro Electrónica. Vol. 2 No. 19. Cuba (Pp. 63-71).

Martínez, Miguel. (2016). Aspectos socioculturales del desarrollo comunitario. Revista Cubana de Ciencias Sociales. Vol. 1 No. 6. Cuba (Pp. 1-12).

Montoro, Ricardo. (1997). Fundamentos teóricos de la política social. Política Social. Vol. 1 No. 1997. España (Pp. 1-17).

Olivera, Dianelys; Peralta, María Josefa y García, Ognara. (2018). La coautoría como expresión de la colaboración en la producción científica de Camagüey. Biblios. Vol. 423 No. 70. Brasil (Pp. 1-16). Doi: https://doi.org/10.5195/biblios.2018.423

Olson, Mark y Fahey, Jay. (2011). Moringa oleifera: un árbol multiusos para las zonas tropicales secas. Revista mexicana de biodiversidad. Vol. 82 No. 4. México (Pp. 1071-1082).

Organización de las Naciones Unidas Para la Alimentación y la Agricultura. (2012). Agronoticias: Actualidad agropecuaria de América Latina y el Caribe. FAO, Estados Unidos.

Pacheco, Tomás. (2006). Aportes de la sociología al estudio de la ciencia como proceso social y producto cultural. Ludus Vitalis. Vol. 14 No. 25. México (Pp. 95-104).

Paz, Luis Ernesto y Caramés, Marialy. (2020). Concepciones para el análisis de campos científicos. TELOS: Revista de Estudios Interdisciplinarios en Ciencias Sociales. Vol. 22 No. 1. Venezuela (Pp. 106-124). Doi: https://doi.org/10.36390/telos221.08

Riera, Celia Marta; Paz, Luis Ernesto y Hernández Alfonso, Eduardo Alejandro. (2018). Consideraciones sobre el desarrollo comunitario. Investigación y Desarrollo. Vol. 26 No. 1. Colombia (Pp. 29-43). 
Rodríguez, Alejandra. (2017). Configuración del campo de la comunicación política: prácticas y redes de investigadores mexicanos. Instituto de Ciencias de Gobierno y Desarrollo Estratégico, México.

Spinak, Ernesto. (1996). Diccionario Enciclopédico de Bibliometría, Cienciometría e Informetría. UNESCO, Cuba. 